ARTICLE

https://doi.org/10.1038/s41467-019-12788-0

\title{
Dopant-tuned stabilization of intermediates promotes electrosynthesis of valuable C3 products
}

Tao-Tao Zhuang 1,2,5, Dae-Hyun Nam (1) 1,5, Ziyun Wang (1) 1,5, Hui-Hui Li,2, Christine M. Gabardo (D) ${ }^{3}$, Yi Li $^{2}$,

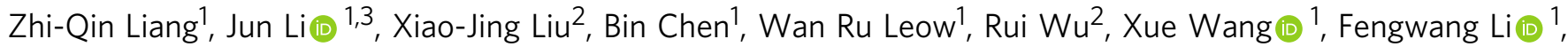
Yanwei Lum¹, Joshua Wicks', Colin P. O'Brien ${ }^{3}$,Tao Peng ${ }^{1}$, Alexander H. Ip ${ }^{1}$, Tsun-Kong Sham ${ }^{4}$,

Shu-Hong Yu (i) ${ }^{2}$, David Sinton (i) $^{3} \&$ Edward H. Sargent (i) ${ }^{1 *}$

The upgrading of $\mathrm{CO}_{2} / \mathrm{CO}$ feedstocks to higher-value chemicals via energy-efficient electrochemical processes enables carbon utilization and renewable energy storage. Substantial progress has been made to improve performance at the cathodic side; whereas less progress has been made on improving anodic electro-oxidation reactions to generate value. Here we report the efficient electroproduction of value-added multi-carbon dimethyl carbonate (DMC) from $\mathrm{CO}$ and methanol via oxidative carbonylation. We find that, compared to pure palladium controls, boron-doped palladium $(\mathrm{Pd}-\mathrm{B})$ tunes the binding strength of intermediates along this reaction pathway and favors DMC formation. We implement this doping strategy and report the selective electrosynthesis of DMC experimentally. We achieve a DMC Faradaic efficiency of $83 \pm 5 \%$, fully a $3 x$ increase in performance compared to the corresponding pure Pd electrocatalyst.

\footnotetext{
${ }^{1}$ Department of Electrical and Computer Engineering, University of Toronto, 35 St George Street, Toronto, ON M5S 1A4, Canada. ${ }^{2}$ Division of Nanomaterials \& Chemistry, Hefei National Laboratory for Physical Sciences at Microscale, Collaborative Innovation Center of Suzhou Nano Science and Technology, CAS Center for Excellence in Nanoscience, Department of Chemistry, University of Science and Technology of China, Hefei, Anhui 230026, P. R. China.

${ }^{3}$ Department of Mechanical and Industrial Engineering, University of Toronto, 5 King's College Road, Toronto, ON M5S 3G8, Canada. ${ }^{4}$ Department of Chemistry, University of Western Ontario, 1151 Richmond Street, London, ON N6A 5B7, Canada. ${ }^{5}$ These authors contributed equally: Tao-Tao Zhuang, DaeHyun Nam, Ziyun Wang. *email: ted.sargent@utoronto.ca
} 
he cathodic carbon dioxide $\left(\mathrm{CO}_{2}\right)$ reduction reaction has seen rapid progress of late, including in the production of $\mathrm{CO}$, methane, formic acid, ethylene, ethanol, and propanol $^{1-8}$. At present, most electrochemical anodic side reactions have utilized the oxygen evolution reaction (OER).

Anodic reactions offer, in principle, valuable upgrades of waste products and lower-value commodity chemicals; yet have seen less exploration in electrocatalysis ${ }^{9-12}$. In anodic chemical upgrade reactions, a particularly important challenge is to achieve selective electro-oxidation to the desired valuable product, instead of overoxidizing the inputs all the way to $\mathrm{CO}_{2}$.

Industrial effluent streams and steel flue gas contain $\mathrm{CO}$, a high-energy feedstock that nonetheless commands a low market value ${ }^{13-15}$. The impressive progress of $\mathrm{CO}_{2}$-to-CO using electrocatalysis further motivates exploring ways to upgrade $\mathrm{CO}$ produced from $\mathrm{CO}_{2}$ to more valuable products.

Here we explore coupling methanol and $\mathrm{CO}$ via electrochemical oxidative carbonylation to dimethyl carbonate ${ }^{16-18}$ (DMC, Eq. (1)). This enables the production of a valuable C3 compound-one already industrially important as a fuel additive, as a polar solvent, and as an environmentally sustainable intermediate for the upgrade of several promising renewables ${ }^{19,20}$. The global market for DMC will exceed $\$ 500$ M USD by $2025^{21}$ and our technoeconomic assessment (TEA, Supplementary Figs. 1, 2, and Table 1) indicates a production cost of US\$1200/ton for DMC from total chemical + renewable electricity inputs to be $\sim$ US\$600/ton.

To catalyze the electrosynthetic pathway

$$
2 \mathrm{CH}_{3} \mathrm{OH}+\mathrm{CO}-2 \mathrm{e}^{-} \rightarrow\left(\mathrm{CH}_{3} \mathrm{O}\right)_{2} \mathrm{CO}+2 \mathrm{H}^{+}
$$

palladium (Pd)-based electrodes achieve methanol carbonylation with $\mathrm{CO}^{22,23}$, but suffer from the formation of by-products, such as dimethyl oxalate, lowering the DMC selectivity.

We first investigated the reaction steps with the goal of further understanding mechanism; and then used these insights to engineer catalysts to increase selectivity to DMC production. We utilized density functional theory (DFT) to explore what controls the binding strength of intermediates on the catalyst along the methanol-CO to DMC pathway. Our findings motivate us to attempt the doping of $\mathrm{Pd}$ to tune intermediate binding energies to favor DMC formation, a strategy we implemented experimentally, allowing us to achieve high-faradaic-efficiency conversion to DMC. This work suggests further potential in interstitial doping to promote oxidation-based carbon upgrade reactions using renewable feedstocks.

\section{Results}

Computational studies. We first investigated, using computational studies, the reaction of methanol-CO to DMC on Pd(111). The DMC formation reaction begins with $\mathrm{CO}$ adsorption to form $\mathrm{CO}^{*}$ (Fig. 1a) and the dissociation of $\mathrm{CH}_{3} \mathrm{OH}$ into $\mathrm{CH}_{3} \mathrm{O}^{*}$ (Fig. 1b). These intermediates undergo coupling to form $\mathrm{CH}_{3} \mathrm{OCO}^{*}$ (Fig. 1c). DMC is then generated through the formation of a $\mathrm{C}-\mathrm{O}$ bond between $\mathrm{CH}_{3} \mathrm{OCO}^{*}$ and another $\mathrm{CH}_{3} \mathrm{O}^{*}$. The dissociation of $\mathrm{CH}_{3} \mathrm{OH}$ is the only step among these that involves electron transfer (implicated in proton coupled electron transfer $)^{24}$. We then evaluated the effect of applied potential on the reaction using the computational hydrogen electrode of Nørskov and co-workers ${ }^{25}$ and applied $1 \mathrm{~V}$ vs. SHE toward DMC electrocatalytic formation ${ }^{16}$.

The energy profile (Fig. 1d and Supplementary Table 2) indicates strong binding of the adsorbed $\mathrm{CO}^{*}$ and the dissociated $\mathrm{CH}_{3} \mathrm{O}^{*}$ on pure $\mathrm{Pd}$. It is so strong as to render further coupling of $\mathrm{CO}^{*}$ and $\mathrm{CH}_{3} \mathrm{O}^{*}$ unfavorable. The barriers associated with the $\mathrm{C}-\mathrm{O}$ bond formation steps including $\mathrm{OC}-\mathrm{OCH}_{3}$ (TS1) and $\mathrm{CH}_{3} \mathrm{O}-\mathrm{C}(\mathrm{O}) \mathrm{OCH}_{3}$ (TS2) are 1.49 and $0.82 \mathrm{eV}$ (Fig. 1d and Supplementary Fig. 4), respectively, indicating that coupling is also unfavorable kinetically. Clearly, tuning the binding strength of the catalyst has the potential to improve DMC formation.

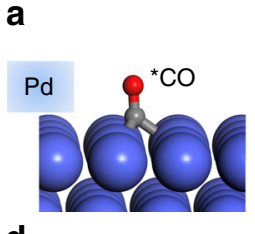

b

d

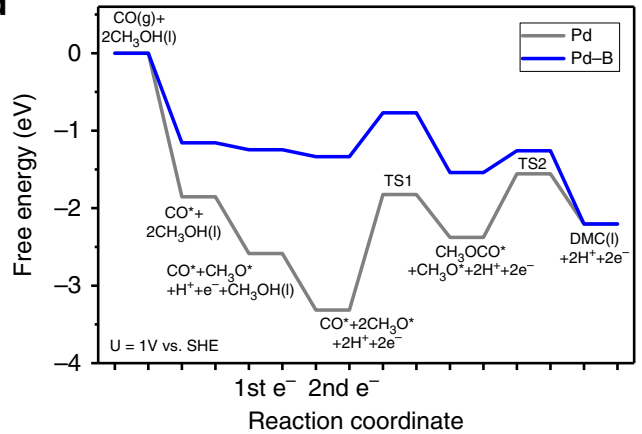

e

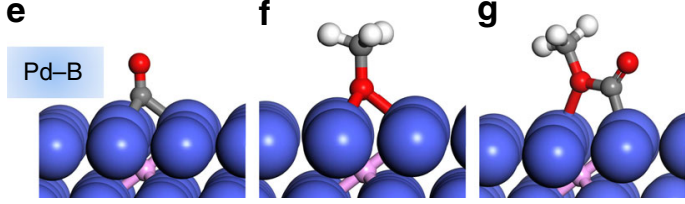

C

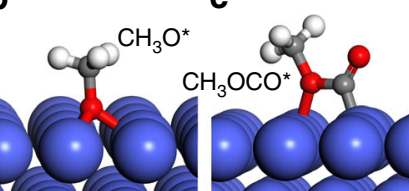

Pd

(1)

$+2 \mathrm{e}^{-}$

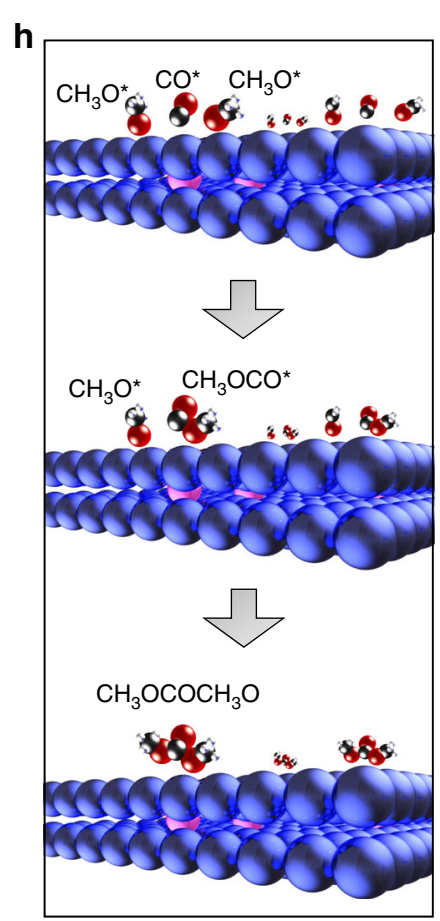

Fig. 1 DFT calculations on DMC formation. Geometries of intermediates in $\mathrm{DMC}$ formation reactions: a $\mathrm{CO}, \mathbf{b} \mathrm{CH}_{3} \mathrm{O}, \mathbf{c} \mathrm{CH}_{3} \mathrm{OCO}$ on pure $\mathrm{Pd}$; e $\mathrm{CO}$, $\mathbf{f} C \mathrm{CH}_{3} \mathrm{O}$, $\mathbf{g ~} \mathrm{CH}_{3} \mathrm{OCO}$ on boron-doped $\mathrm{Pd}\left(\mathrm{Pd}-\mathrm{B}\right.$ ). $\mathbf{d}$ Free energy profiles of $\mathrm{DMC}$ formation from $\mathrm{CO}$ and $\mathrm{CH}_{3} \mathrm{OH}$ on pure $\mathrm{Pd}$ (gray) and $\mathrm{Pd}-\mathrm{B}$ (blue) at $\mathrm{U}=1 \mathrm{~V}$ vs. SHE. h Reaction scheme for electroproduction of DMC from methanol and $\mathrm{CO}$ on the Pd-B electrode. $\mathrm{DMC} / \mathrm{CH}_{3} \mathrm{OCOCH} \mathrm{H}_{3} \mathrm{O}$ : dimethyl carbonate. Red, gray, white, pink, and blue balls represent oxygen, carbon, hydrogen, boron, and palladium, respectively 

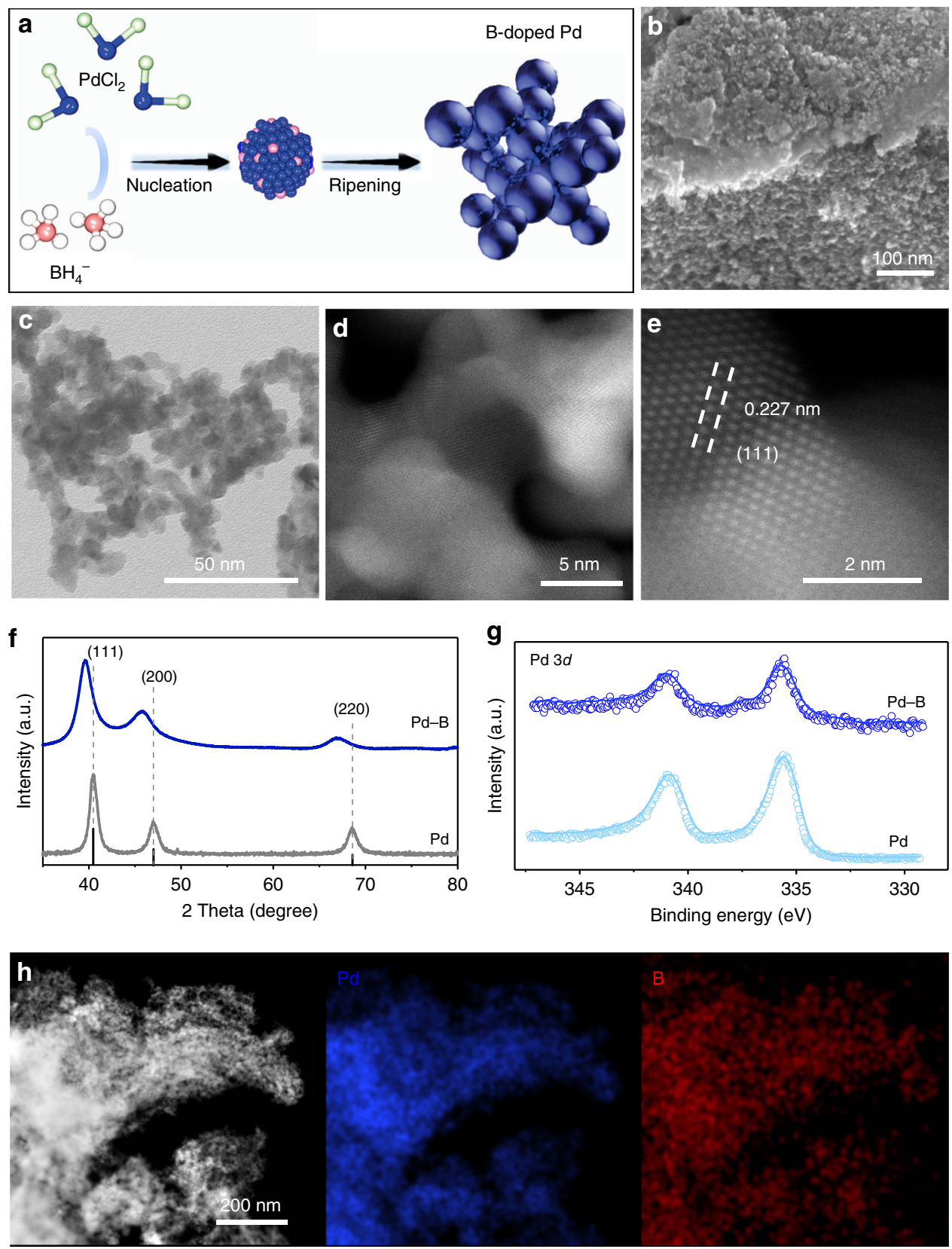

Fig. 2 Catalyst synthesis and structure characterization. a The fabrication schematic illustration of Pd-B catalysts. b SEM, c TEM, d HAADF, and e HAADFSTEM images, showing the morphology and size of Pd-B nanomaterials. $\mathbf{f}$ Powder XRD spectra of Pd-B and Pd samples show a peak shift, demonstrating the boron penetration into the Pd lattice. Black line corresponding to JCPDS No. 05-0681. $\mathbf{g}$ XPS spectra for Pd 3d regions. $\mathbf{h}$ EELS, showing the homogeneous distribution of Pd and B elements in the Pd-B sample. The scale bar in $\mathbf{h}$ is $200 \mathrm{~nm}$

We investigated boron doping of $\mathrm{Pd}$ in the context of the anodic oxidative carbonylation reaction (Fig. 1e-g, Supplementary Fig. 3 and Supplementary Tables 3-5). The results (Fig. 1d) reveal that boron doping controls the adsorption of both $\mathrm{CO}^{*}$ and $\mathrm{CH}_{3} \mathrm{O}^{*}$, rendering the energies of intermediates along the reaction process downhill toward $\mathrm{CH}_{3} \mathrm{OCO}^{*}$ formation. Furthermore, the barriers are $0.57 \mathrm{eV}$ for TS1 and $0.28 \mathrm{eV}$ for TS2 on boron-doped palladium (Pd-B), which are significantly lower compared to those on Pd (Fig. 1d), thus indicating increased selectivity to DMC electrosynthesis on Pd-B (Fig. 1h).

Catalyst synthesis and characterization. Experimentally we prepare $\mathrm{Pd}-\mathrm{B}$ interstitial nanoalloys (Fig. 2a, details in the "Methods" section). We used scanning electron microscopy
(SEM), transmission electron microscopy (TEM), and high-angle annular dark-field scanning transmission electron microscopy (HAADF-STEM) (Fig. 2b-d) to examine the morphology and size of the resultant Pd-B. We obtained a dendritic Pd-B morphology comprised of nanoparticles ranging in diameter from 5 to $10 \mathrm{~nm}$.

From TEM, the Pd-B lattice spacing is $0.227 \mathrm{~nm}$ (Fig. 2e), larger than that of pure Pd $(0.222 \mathrm{~nm}$, Supplementary Fig. 5), consistent with lattice expansion when $\mathrm{B}$ atoms penetrate into the Pd lattice. Powder X-ray diffraction (PXRD) confirms the same trend: the diffraction peak of $\mathrm{Pd}-\mathrm{B}$ shifts to a lower $2 \theta$ value compared to that of the Pd control (Fig. 2f) 26,27 . X-ray photoelectron spectroscopy (XPS) confirms the change in the electronic structure of Pd via B-doping as seen in the slight shift 
a

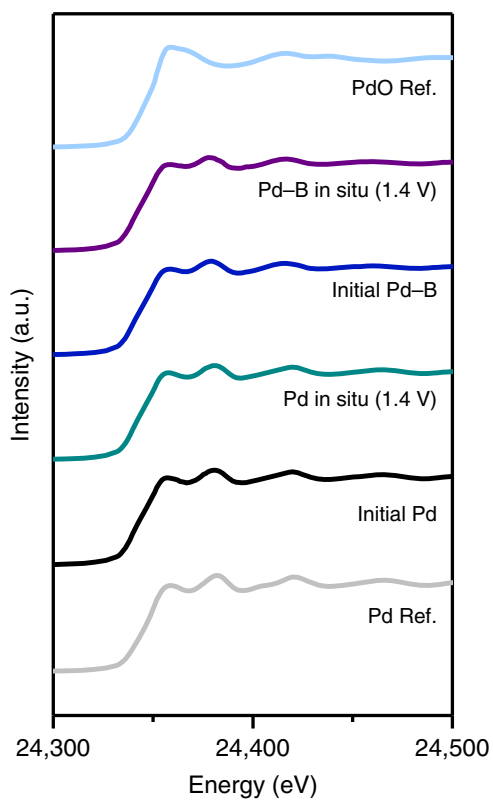

b

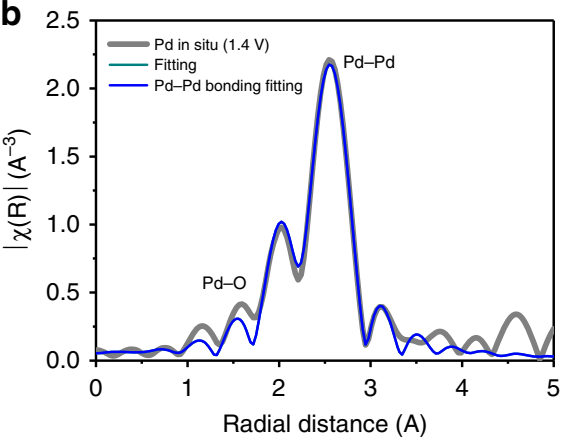

d

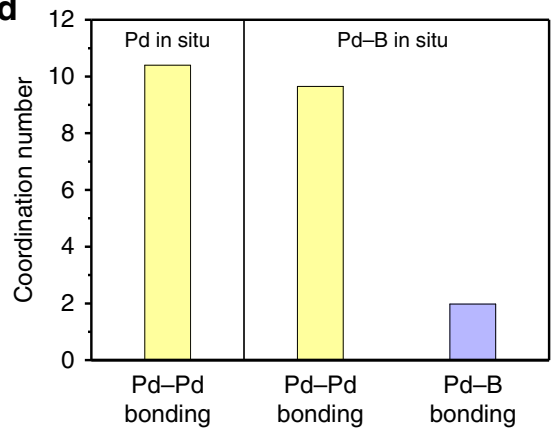

C
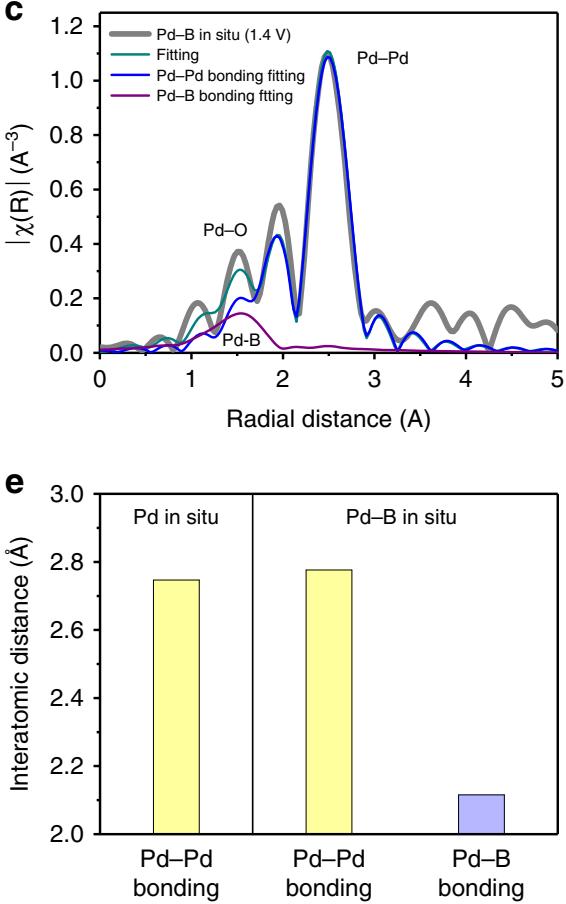

Fig. 3 Operando XAS of catalysts during DMC electroproduction. a Pd K-edge XAS for investigating the Pd and Pd-B catalysts at the potential of $1.4 \mathrm{~V}$ vs. $\mathrm{Ag} / \mathrm{AgCl}$. b, $\mathbf{c}$ Operando EXAFS of (b) Pd and (c) Pd-B to investigate the local atomic structure around the Pd atom with Pd-Pd and Pd-B fitting. d, e EXAFS fitting induced (d) coordination number and (e) interatomic distance comparison between $\mathrm{Pd}$ and $\mathrm{Pd}-\mathrm{B}$

of Pd 3d-binding energy (Fig. 2g), while electron energy loss spectroscopy (EELS) mapping reveals that $\mathrm{Pd}$ and $\mathrm{B}$ are uniformly distributed in the Pd-B sample (Fig. 2h).

We tuned the $\mathrm{B}$ concentration inside the Pd nanocrystals by varying the precursor ratio between $\mathrm{Pd}$ and B (see the "Methods" section). The sample retained a similar size and shape as we increased B content, and lattice fringes expanded and the diffraction peak shifted further (Supplementary Figs. 6 and 7).

We then investigated, using operando X-ray absorption spectroscopy $^{28}$ (XAS), the stability of $\mathrm{Pd}-\mathrm{B}$ during $\mathrm{DMC}$ electroproduction, investigating both pure $\mathrm{Pd}$ and $\mathrm{Pd}-\mathrm{B}$ electrocatalysts (Fig. 3). We probed the local environment in the vicinity of the Pd atoms by tracking changes in X-ray absorption near edge structure (XANES) and extended X-ray absorption fine structure (EXAFS) spectra. These were measured during anodic reaction under a potential of $1.4 \mathrm{~V}$ vs. $\mathrm{Ag} / \mathrm{AgCl}$ in $0.1 \mathrm{M} \mathrm{NaClO}_{4} /$ methanol electrolyte with continuous $\mathrm{CO}$ gas flowing.

$\mathrm{Pd}$ K-edge XANES of pure $\mathrm{Pd}$ and $\mathrm{Pd}-\mathrm{B}$ electrocatalysts revealed no evident change in $\mathrm{Pd}$ valence state during the reaction (Fig. 3a). We found that the electrode composition was stable across the reaction time (XPS, Supplementary Fig. 8). When we compared the average oxidation state of $\mathrm{Pd}$ at $1.4 \mathrm{~V}$ vs. $\mathrm{Ag} / \mathrm{AgCl}$, the XANES linear combination fitting of Pd-B resulted in +0.68 as an average oxidation state, which is higher than the average oxidation state of pure Pd, +0.59 (Supplementary Table 6). Both pure $\mathrm{Pd}$ and $\mathrm{Pd}-\mathrm{B}$ showed lower oxidation states than the +2 of $\mathrm{PdO}$. We attribute the higher $\mathrm{Pd}$ oxidation state of $\mathrm{Pd}-\mathrm{B}$ to bonding between $\mathrm{Pd}$ and $\mathrm{B}$.

Using EXAFS to acquire information on atomic bonding near the $\mathrm{Pd}$ atom (Fig. $3 \mathrm{~b}$ and $\mathrm{c}$ ), we found that $\mathrm{Pd}-\mathrm{B}$ has a lower $\mathrm{Pd}-\mathrm{Pd}$ coordination number (Pd: 10.4, Pd-B: 9.7) and longer Pd-Pd interatomic distance (Pd: $2.747 \AA$, Pd-B: $2.776 \AA$ ) compared to that of pure Pd (Fig. $3 \mathrm{~d}$, e) during electrocatalytic DMC production when B is present (Supplementary Fig. 9). Pd-B fitting of EXAFS (Pd-B coordination number: 1.98, $\mathrm{Pd}-\mathrm{B}$ interatomic distance: $2.115 \AA$ ) indicates the same trend. We conclude that the interstitial $\mathrm{B}$ doping in the Pd lattice is stable across reaction times studied herein.

Electrochemical oxidation reaction investigations. We deposited catalysts onto carbon paper via spray coating (see the "Methods" section) and characterized the electrochemical CO-methanol oxidative carbonylation activity and selectivity toward DMC using a three-electrode $\mathrm{H}$-cell configuration. We first measured cyclic voltammograms (CV) of the anodes to study electrocatalytic carbonylation. In nitrogen-purged electrolytes $\left(0.1 \mathrm{M} \mathrm{NaClO}_{4} / \mathrm{methanol}\right.$, we observed a broad oxidation peak $(\mathrm{Ox}-1)$ for all $\mathrm{Pd}-\mathrm{B}$ samples at ca. $1.2 \mathrm{~V}$ that we ascribe to the electrochemical oxidation of $\mathrm{Pd}^{0}$ to $\mathrm{Pd}^{2+}$ with methanol (Eq. (2), Fig. 4a left, and Supplementary Fig. 10) ${ }^{23}$. A steep increase in current was seen at potentials higher than $1.5 \mathrm{~V}(\mathrm{Ox}-2)$ owing to direct methanol oxidation (Eqs. (3) and (4) $)^{22}$. Upon bubbling and saturation of the solution with $\mathrm{CO}$, the oxidative insertion of $\mathrm{CO}$ into methanol occurred (broad oxidation peak at ca. $1.5 \mathrm{~V}$, Ox-3) 29 . This implied DMC formation (Fig. 4b), and the product was further evaluated by gas chromatography with flameionization detection and gas chromatography with mass spectrometry (GC-FID and GC-MS, Supplementary Figs. 11 and 12), respectively. CO-methanol oxidative carbonylation suppressed the Pd electrode self-oxidation evidenced by XAS data in Fig. 3a, and it also shifted the large current of direct methanol oxidation to more positive potentials $(>1.8 \mathrm{~V})$.

$$
\begin{gathered}
\mathrm{Pd}^{0}+2 \mathrm{CH}_{3} \mathrm{OH} \rightarrow \mathrm{Pd}^{2+}\left(\mathrm{CH}_{3} \mathrm{O}^{-}\right)_{2}+2 \mathrm{H}^{+} \\
3 \mathrm{CH}_{3} \mathrm{OH}-2 \mathrm{e}^{-} \rightarrow\left(\mathrm{CH}_{3} \mathrm{O}\right)_{2} \mathrm{CH}_{2}+2 \mathrm{H}^{+}+\mathrm{H}_{2} \mathrm{O} \\
2 \mathrm{CH}_{3} \mathrm{OH}-4 \mathrm{e}^{-} \rightarrow \mathrm{HC}(\mathrm{O}) \mathrm{OCH}_{3}+4 \mathrm{H}^{+}
\end{gathered}
$$

We then evaluated the CO-methanol oxidation performance in the potential range of $1.0-1.6 \mathrm{~V}$ versus $\mathrm{Ag} / \mathrm{AgCl}(\mathrm{CO}-m e t h a n o l$ 
a

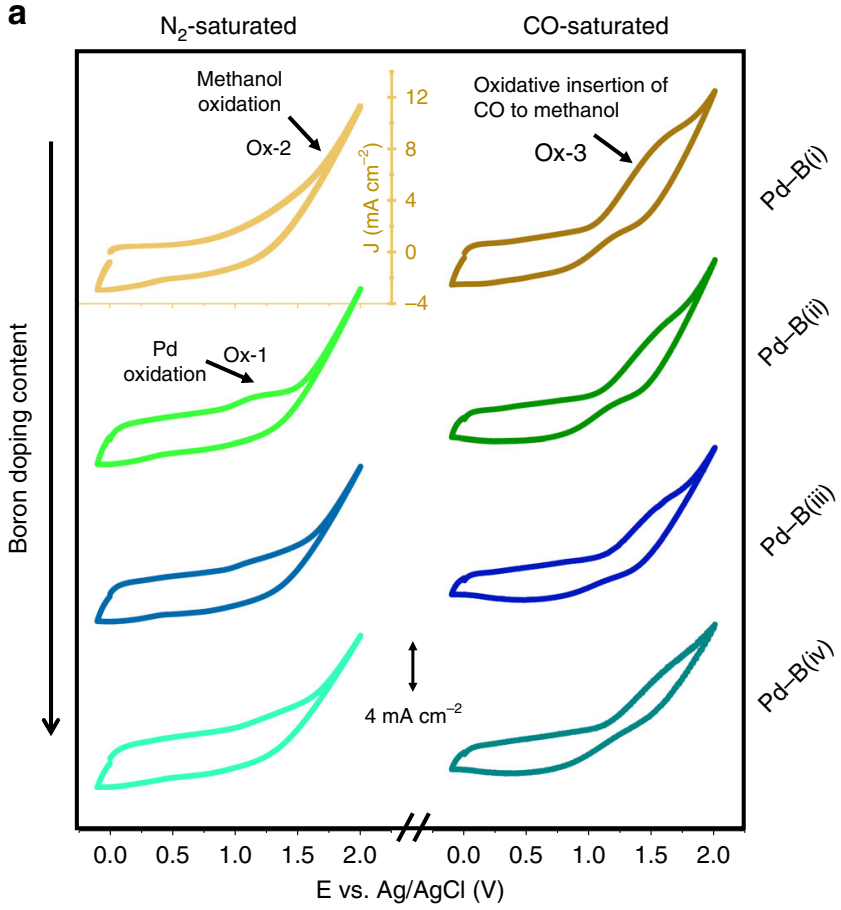

b

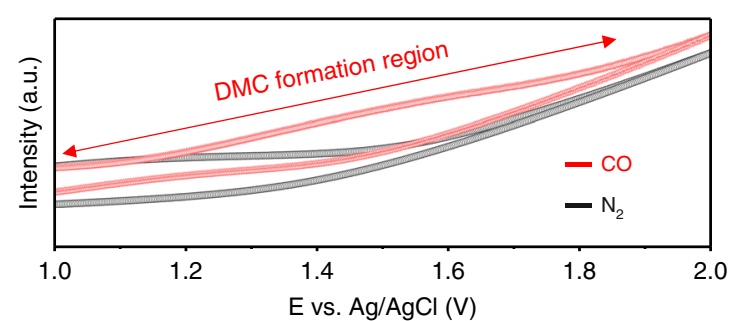

C

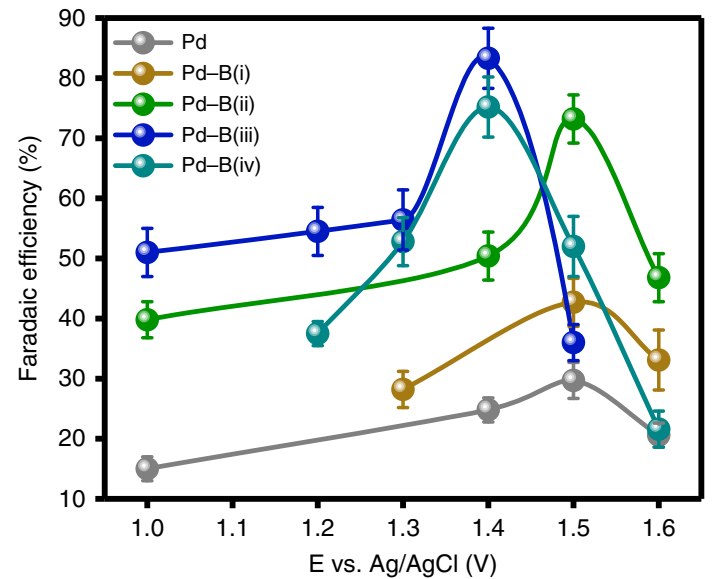

d

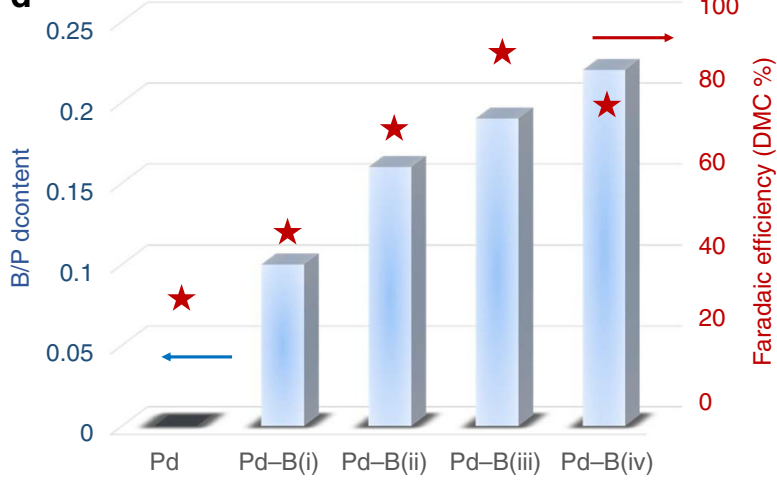

Fig. 4 Effects of boron doping in palladium on the DMC electroproduction. a Cyclic voltammograms on the Pd-B electrodes, $0.1 \mathrm{M} \mathrm{NaClO} 4 / \mathrm{methanol}$ electrolytes; scan rate $50 \mathrm{mV} \mathrm{S}^{-1}$. Left: $\mathrm{N}_{2}$-saturated; right: CO-saturated. $\mathbf{b}$ amplified $\mathrm{CV}$, highlighting DMC formation region. $\mathbf{c}$ DMC Faradaic efficiencies on the catalysts under different applied potentials show the Pd-B (iii) sample has the best selectivity. $\mathbf{d}$ The effect of B doping content in Pd on DMC selectivity. Error bars correspond to the standard deviation of three or more measurements

coupling region) in $0.1 \mathrm{M} \mathrm{NaClO} /$ methanol. In this way we would investigate further the effect of the $\mathrm{B}$ dopant on DMC selectivity. Compared to pure $\mathrm{Pd}$, all $\mathrm{Pd}-\mathrm{B}$ samples showed higher DMC selectivity (Fig. $4 \mathrm{c}$ and Supplementary Table 8 ). At $1.4 \mathrm{~V}$ vs. $\mathrm{Ag} / \mathrm{AgCl}$, we achieved Faradaic efficiency of $83 \pm 5 \%$ for DMC using the $\mathrm{Pd}-\mathrm{B}$ (iii) catalyst.

This DMC production represents a three-fold improvement in selectivity compared to unmodified Pd (Supplementary Table 8 and 9). We analyzed the B dopant amount through inductively coupled plasma mass spectrometry (ICP-MS, Supplementary Table 10) and report as a result the $\mathrm{B}$ doping present in the catalyst (Fig. 4d) and correlate this descriptor with high DMC electrosynthesis.

\section{Discussion}

Selective electroproduction of the C3 liquid chemical DMC from lower-value $\mathrm{CO}$ and methanol was achieved by oxidative carbonylation with the aid of a new boron-doped palladium electrocatalyst. Boron improved DMC selectivity verified through DFT calculations, material structure analysis, and electrochemical measurements. This work offers an avenue to upgrade carbon via electro-oxidation that could be applied to electrolyzers to achieve high-value products from both the cathodic and the anodic reactions. Ultimately, tandem electrocatalytic cathode-anode systems uniting $\mathrm{CO}_{2}$-to- $\mathrm{CO}$ (cathodic reduction) with $\mathrm{CO}$-toDMC (anodic oxidation) stand to offer integrated DMC production from $\mathrm{CO}_{2}$.

\section{Methods}

DFT calculations. In this work, all DFT calculations were carried out with a periodic slab model using the Vienna ab initio simulation program (VASP) ${ }^{30}$ (https://www.vasp.at/). Detailed theoretical methods can be found in Supplementary Methods.

Catalyst synthesis. $\mathrm{Pd}-\mathrm{B}$ nanoparticles ${ }^{31}$ were prepared via the rapid chemical reduction reaction between palladium chloride $\left(\mathrm{PdCl}_{2}\right.$, Sigma-Aldrich) and sodium borohydride $\left(\mathrm{NaBH}_{4}\right.$, Sigma-Aldrich). $\mathrm{PdCl}_{2}(89 \mathrm{mg})$ was dissolved in $2.5 \mathrm{~mL}$ deionized (DI) water. The $\mathrm{B}$ dopant concentration was controlled by dissolving $\mathrm{NaBH}_{4}$ in $12.5 \mathrm{~mL}$ DI water $(62.5,250,500,1000 \mathrm{mg}) . \mathrm{NaBH}_{4}$ solution was placed in $\mathrm{PdCl}_{2}$ solution. After the reaction between $\mathrm{PdCl}_{2}$ and $\mathrm{NaBH}_{4}$, the $\mathrm{Pd}-\mathrm{B}$ nanoparticles were washed using DI water. After centrifuging, $\mathrm{Pd}-\mathrm{B}$ nanoparticles were dried in a vacuum oven overnight. Pure Pd nanoparticles were prepared using hydrazine $\left(\mathrm{N}_{2} \mathrm{H}_{4}\right.$, Sigma-Aldrich) as a reducing agent for $\mathrm{PdCl}_{2}$ instead of $\mathrm{NaBH}_{4}$.

Working electrode preparation and oxidation measurements. To prepare electrodes, we deposited $10 \mathrm{mg}$ of catalyst mixed with $20 \mu \mathrm{l}$ of $5 \mathrm{wt} \%$ Nafion in 
$1 \mathrm{~mL}$ methanol on a carbon gas-diffusion layer with loading ca. $1 \mathrm{mg} \mathrm{cm}^{-2}$ using an air-brush spray coater.

Electrocatalytic measurements were carried out in a three-electrode system using an electrochemical station (PGSTAT204, Metrohm Autolab). Electrolysis was carried out in a two-compartment electrochemical $\mathrm{H}$-cell with a proton exchange membrane (Nafion 117) as the separator. All potentials were measured against a $\mathrm{Ag} / \mathrm{AgCl}$ reference electrode $(3 \mathrm{M} \mathrm{KCl}, \mathrm{BASi}$ ) and a platinum counter electrode. In the $\mathrm{H}$-cell, the electrolyte was $0.1 \mathrm{M} \mathrm{NaClO}_{4} /$ methanol saturated with $\mathrm{CO}$, which was delivered into the anodic compartment at a rate of 30.00 standard cubic centimeters per minute (s.c.c.m.).

Dimethyl carbonate analysis. The reacted solution was collected and quantitative analysis. The dimethyl carbonate (DMC) product was carried out using a capillary gas chromatograph (PerkinElmer Clarus 580 and Clarus SQ 8C with FID and MS detectors, respectively) with Stabilwax column (fused silica, Restek). The Faradaic efficiency (FE) of DMC was calculated from the total amount of charge $Q$ (in units of C) passed through the sample and the total amount of the DMC produced $n$ (in moles). $Q=I \times t$, where $I$ (in amperes) is the oxidation current at a specific applied potential and $t$ is the time (in seconds) for the constant oxidation current.

The FE of the DMC is calculated as follows:

$$
\mathrm{FE}_{\mathrm{DMC}}=2 \times F \times \frac{n_{\mathrm{DMC}}}{\mathrm{Q}} \times 100 \%=2 \times F \times \frac{n_{\mathrm{DMC}}}{(I \times t)} \times 100 \%
$$

\section{Data availability}

The data that support the findings of this study are available from the corresponding author on reasonable request.

Received: 19 May 2019; Accepted: 27 September 2019; Published online: 22 October 2019

\section{References}

1. Raciti, D., Livi, K. J. \& Wang, C. Highly dense Cu nanowires for lowoverpotential $\mathrm{CO}_{2}$ reduction. Nano Lett. 15, 6829-6835 (2015).

2. Luc, W. et al. Ag-Sn bimetallic catalyst with a core-shell structure for $\mathrm{CO}_{2}$ reduction. J. Am. Chem. Soc. 139, 1885-1893 (2017).

3. Weekes, D. M., Salvatore, D. A., Reyes, A., Huang, A. \& Berlinguette, C. P. Electrolytic $\mathrm{CO}_{2}$ reduction in a flow cell. Acc. Chem. Res. 51, 910-918 (2018).

4. Nielsen, D. U., Hu, X. M., Daasbjerg, K. \& Skrydstrup, T. Chemically and electrochemically catalysed conversion of $\mathrm{CO}_{2}$ to $\mathrm{CO}$ with follow-up utilization to value-added chemicals. Nat. Catal. 1, 244-254 (2018).

5. Dinh, C. T. et al. $\mathrm{CO}_{2}$ electroreduction to ethylene via hydroxide-mediated copper catalysis at an abrupt interface. Science 360, 783-787 (2018).

6. Li, Y. C. et al. Binding site diversity promotes $\mathrm{CO}_{2}$ electroreduction to ethanol. J. Am. Chem. Soc. 141, 8584-8591 (2019).

7. Zhuang, T. T. et al. Copper nanocavities confine intermediates for efficient electrosynthesis of C3 alcohol fuels from carbon monoxide. Nat. Catal. 1, 946-951 (2018).

8. Liang, Z. Q. et al. Copper-on-nitride enhances the stable electrosynthesis of multi-carbon products from $\mathrm{CO}_{2}$. Nat. Commun. 9, 3828 (2018).

9. Zhang, B. et al. Homogeneously dispersed multimetal oxygen-evolving catalysts. Science 352, 333-337 (2016).

10. Li, H.-H. et al. Highly crystalline PtCu nanotubes with three dimensional molecular accessible and restructured surface for efficient catalysis. Energy Environ. Sci. 10, 1751-1756 (2017).

11. Ma, S.-Y. et al. Synthesis of low Pt-based quaternary PtPdRuTe nanotubes with optimized incorporation of Pd for enhanced electrocatalytic activity. J. Am. Chem. Soc. 139, 5890-5895 (2017).

12. Zheng, X. et al. Theory-driven design of high-valence metal sites for water oxidation confirmed using in situ soft X-ray absorption. Nat. Chem. 10, 149 (2018).

13. Verma, S., Lu, X., Ma, S., Masel, R. I. \& Kenis, P. J. The effect of electrolyte composition on the electroreduction of $\mathrm{CO}_{2}$ to $\mathrm{CO}$ on $\mathrm{Ag}$ based gas diffusion electrodes. Phys. Chem. Chem. Phys. 18, 7075-7084 (2016).

14. Jhong, H. R. M. et al. A nitrogen-doped carbon catalyst for electrochemical $\mathrm{CO}_{2}$ conversion to $\mathrm{CO}$ with high selectivity and current density. ChemSusChem 10, 1094-1099 (2017).

15. Gabardo, C. M. et al. Combined high alkalinity and pressurization enable efficient $\mathrm{CO}_{2}$ electroreduction to CO. Energy Environ. Sci. 11, 2531-2539 (2018).

16. Figueiredo, M. C., Trieu, V., Eiden, S. \& Koper, M. T. Spectro-electrochemical examination of the formation of dimethyl carbonate from $\mathrm{CO}$ and methanol at different electrode materials. J. Am. Chem. Soc. 139, 14693-14698 (2017).

17. Davies, B. J. et al. Electrochemically generated copper carbonyl for selective dimethyl carbonate synthesis. ACS Catal. 9, 859-866 (2018).
18. Tan, H.-Z. et al. Review on the synthesis of dimethyl carbonate. Catal. Today 316, 2-12 (2018)

19. Tundo, P. \& Selva, M. The chemistry of dimethyl carbonate. Acc. Chem. Res. 35, 706-716 (2002)

20. Fiorani, G., Perosa, A. \& Selva, M. Dimethyl carbonate: a versatile reagent for a sustainable valorization of renewables. Green Chem. 20, 288-322 (2018)

21. Outcalt, S. L. \& Laesecke, A. Compressed-liquid densities of the binary mixture dimethyl carbonate + heptane at three compositions. J. Mol. Liq. 279, 378-385 (2019).

22. Yamanaka, I., Funakawa, A. \& Otsuka, K. Selective carbonylation of methanol to dimethyl carbonate by gas-liquid-solid-phase boundary electrolysis. Chem. Lett. 31, 448-449 (2002).

23. Yamanaka, I., Funakawa, A. \& Otsuka, K. Electrocatalytic synthesis of DMC over the Pd/VGCF membrane anode by gas-liquid-solid phase-boundary electrolysis. J. Catal. 221, 110-118 (2004).

24. Huynh, M. H. V. \& Meyer, T. J. Proton-coupled electron transfer. Chem. Rev. 107, 5004-5064 (2007)

25. Nørskov, J. K. et al. Origin of the overpotential for oxygen reduction at a fuelcell cathode. J. Phys. Chem. B 108, 17886-17892 (2004).

26. Chan, C. W. A. et al. Interstitial modification of palladium nanoparticles with boron atoms as a green catalyst for selective hydrogenation. Nat. Commun. $\mathbf{5}$ 5787 (2014)

27. Vo Doan, T. T. et al. Frontispiece: theoretical modelling and facile synthesis of a highly active boron-doped palladium catalyst for the oxygen reduction reaction. Angew. Chem. Int. Ed. 55, 6842-6847 (2016).

28. Li, J. et al. Copper adparticle enabled selective electrosynthesis of n-propanol. Nat. Commun. 9, 4614 (2018)

29. Funakawa, A., Yamanaka, I., Takenaka, S. \& Otsuka, K. Selectivity control of carbonylation of methanol to dimethyl oxalate and dimethyl carbonate over gold anode by electrochemical potential. J. Am. Chem. Soc. 126, 5346-5347 (2004).

30. Kresse, G. \& Furthmüller, J. Efficient iterative schemes for ab initio totalenergy calculations using a plane-wave basis set. Phys. Rev. B 54, 11169-11186 (1996).

31. Chen, A. \& Ostrom, C. Palladium-based nanomaterials: synthesis and electrochemical applications. Chem. Rev. 115, 11999-12044 (2015)

\section{Acknowledgements}

This work was supported by the Ontario Research Fund Research-Excellence Program, the Natural Sciences and Engineering Research Council (NSERC) of Canada, and the CIFAR Bio-Inspired Solar Energy program. The authors thank Dr. Z. Finfrock and Dr. D.M. Meira for technical support at the 20BM beam-line of the Advanced Photon Source (Lemont, IL). This research used resources of the Advanced Photon Source, an Office of Science User Facility operated for the U.S. Department of Energy (DOE) Office of Science by Argonne National Laboratory, and was supported by the U.S. DOE under Contract No. DE-AC02-06CH11357, and the Canadian Light Source and its funding partners. S.-H.Y. acknowledges funding from the National Natural Science Foundation of China (Grant 21431006), the Foundation for Innovative Research Groups of the National Natural Science Foundation of China (Grant 21521001). D.S. acknowledges the NSERC E.W.R Steacie Memorial Fellowship. J.L. acknowledges the Banting Postdoctoral Fellowships program. C.M.G acknowledges the NSERC Postdoctoral Fellowships program. All DFT computations were performed on the IBM BlueGene/Q supercomputer with support from the Southern Ontario Smart Computing Innovation Platform (SOSCIP) and Niagara supercomputer at the SciNet HPC Consortium. SOSCIP is funded by the Federal Economic Development Agency of Southern Ontario, the Province of Ontario, IBM Canada Ltd., Ontario Centres of Excellence, Mitacs and 15 Ontario academic member institutions. SciNet is funded by: the Canada Foundation for Innovation; the Government of Ontario; Ontario Research Fund - Research Excellence; and the University of Toronto. The authors thank C.D. and Y.W. from $\mathrm{U}$ of $\mathrm{T}$ for fruitful discussions The authors thank J.X. from Tianjin University of Technology for HAADF-STEM and EELS measurement support.

\section{Author contributions}

E.H.S. supervised the project. T.-T.Z. and E.H.S. conceived the idea and wrote the paper. T.-T.Z. and D.-H.N. designed the structures and carried out the experiments. Z.W. carried out the DFT simulations. Z.-Q.L., Y.L., B.C. and R.W. helped to characterize the morphology of catalyst. D.-H.N., Y.W.L. and J.L. performed the X-ray spectroscopy measurements. H.-H.L., C.M.G., X.-J.L., W.R.L., X.W., F.L., J.W., C.P.O., T.P., A.H.I., T.-K.S., S.-H.Y. and D.S. helped data analysis and manuscript polishing. All authors discussed the results and assisted during manuscript preparation.

\section{Competing interests}

The authors declare no competing interests. 


\section{Additional information}

Supplementary information is available for this paper at https://doi.org/10.1038/s41467019-12788-0.

Correspondence and requests for materials should be addressed to E.H.S.

Peer review information Nature Communications thanks anonymous reviewers for their contributions to the peer review of this work.

Reprints and permission information is available at http://www.nature.com/reprints

Publisher's note Springer Nature remains neutral with regard to jurisdictional claims in published maps and institutional affiliations. (c) (i) Open Access This article is licensed under a Creative Commons Attribution 4.0 International License, which permits use, sharing, adaptation, distribution and reproduction in any medium or format, as long as you give appropriate credit to the original author(s) and the source, provide a link to the Creative Commons license, and indicate if changes were made. The images or other third party material in this article are included in the article's Creative Commons license, unless indicated otherwise in a credit line to the material. If material is not included in the article's Creative Commons license and your intended use is not permitted by statutory regulation or exceeds the permitted use, you will need to obtain permission directly from the copyright holder. To view a copy of this license, visit http://creativecommons.org/ licenses/by/4.0/.

(C) The Author(s) 2019 